\title{
Gravitating (bi-)sphalerons
}

\author{
Y. Brihaye and M. Desoil \\ Faculté des Sciences, Université de Mons-Hainaut, B-7000 Mons, Belgium
}

(December 24, 2018)

\begin{abstract}
The standard model of electroweak interactions is minimally coupled to gravity and the response of the spherically symmetric solutions -the sphaleron and the bisphaleron- to gravity is emphasized. For a given value of the Higgs mass $M_{H}$, several branches of solutions exist which terminate into cusp-catastrophy at some $\left(M_{H}\right.$-depending) critical value of the parameter $\alpha$ defined by the ratio of the vector-boson mass to the Planck mass. A given branch either bifurcates from another one at an intermediate value of $\alpha$ or persists in the limit $\alpha \rightarrow 0$ where it terminates into a flat sphaleron or bisphaleron or into a Bartnik-McKinnon solution. These bifurcation patterns are studied in some details.
\end{abstract}

\section{INTRODUCTION}

The coupling of non-abelian field theories to Einstein gravity constitutes natural extensions of the EinsteinMaxwell equations. One of the surprising issues of such possibilities has been the discovery of regular, finite energy classical solutions in the Einstein-Yang-Mills equations : the series of Bartnik-Mckinnon 11 solutions.

On the other hand the Weinberg-Salam model theory, which couples the $\mathrm{SU}(2) \otimes \mathrm{U}(1)$ Yang-Mills theory to a doublet of complex Higgs fields emerges more and more as the theory of the unified weak and electromagnetic forces. This particular lagrangian is a member of the family of Yang-Mills-Higgs models among which the GeorgiGlashow model is another distinguished example.

One interesting property of the Weinberg-Salam lagrangian is that it admits an unstable, finite energy classical solution called the sphaleron [2]. Generally, sphaleron solutions are expected to play a role in the understanding of baryon non conserving phenomenon which are allowed to take place in the Weinberg-Salam model [3]. When the parameter determining the mass of the Higgs field increases, additional solutions, the bisphalerons, bifurcate from the sphaleron [4,5]. This feature seems to be related to the underlying non-linear character of the classical equations and to the spontaneous breakdown of the symmetry [6 8].

It is therefore natural to couple the Weinberg-Salam model to the Einstein-Hilbert gravitational lagrangian and to study the response to gravity of the classical solutions available in the flat limit. This problem has been partly investigated in [9] and more recently in a report on the aspects of Einstein-Yang-Mills-Higgs equations [10] but it was not treated in details. In particular the response of the bisphaleron solution to gravity was not investigated. In this paper, we reconsider the classical equations of the Einstein-Weinberg-Salam model for spherically symmetric fields (we assume the Weinberg angle, related to the U(1) part of the gauge group, to be zero). Then the gravitating sphaleron and bisphaleron are constructed and assemble into branches of solutions which evolve with $\alpha$, the ratio of the vector-boson mass to the Planck mass. The evolution of the different solutions as functions of $\alpha$ and $M_{H}$ are studied in details.

\section{THE EQUATIONS}

We consider the gauge theory for an SU(2)-Higgs doublet minimally coupled to the Einstein-Hilbert gravitational lagrangian :

$$
L=\sqrt{-g}\left[L_{G}+L_{M}\right]
$$

with

$$
\begin{gathered}
L_{G}=\frac{1}{16 \pi G} R \\
L_{M}=-\frac{1}{4} F_{\mu \nu}^{a} F^{a \mu \nu}+\left(D_{\mu} \Phi\right)^{\dagger}\left(D^{\mu} \Phi\right)-\frac{\lambda}{4}\left(\Phi^{\dagger} \Phi-\frac{v^{2}}{2}\right)^{2}
\end{gathered}
$$


and with the usual definitions for the fields strenghts and covariant derivatives

$$
\begin{gathered}
F_{\mu \nu}^{a}=\partial_{\mu} A_{\nu}^{a}-\partial_{\nu} A_{\mu}^{a}+g \epsilon_{a b c} A_{\mu}^{b} A_{\nu}^{c} \\
D_{\mu} \Phi=\partial_{\mu} \Phi+g\left(A_{\mu}^{a} \sigma_{a}\right) \Phi
\end{gathered}
$$

The matter part $L_{M}$ of this Lagrangian approximates the Weinberg-Salam model of electroweak interactions in the limit of vanishing Weinberg angle $\theta_{W}$ (i.e. the gauge group $\mathrm{SU}(2) \times \mathrm{U}(1)$ is restricted to $\mathrm{SU}(2)$ ).

Here we will study the classical, spherically symmetric solutions of the Lagrangian $L$. In this purpose, we employ the Schwarzschild-like coordinates for the metric

$$
d s^{2}=-A^{2} N d t^{2}+N^{-1} d r^{2}+r^{2}\left(d \theta^{2}+\sin ^{2} \theta d \phi^{2}\right),
$$

and we introduce, as usual, the mass function $m(r)$ by means of

$$
N(r)=1-\frac{2 m(r)}{r} .
$$

Then we use the standard spherically symmetric ansatz for the spatial components of the gauge field (fields are static and $A_{0}=0$ ) and for the Higgs fields (the notations of [11] are used)

$$
A_{i}^{a}=\frac{1-f_{A}(r)}{g r} \epsilon_{a i j} \hat{x}_{j}+\frac{f_{B}(r)}{g r}\left(\delta_{i a}-\hat{x}_{i} \hat{x}_{a}\right)+\frac{f_{C}(r)}{g r} \hat{x}_{i} \hat{x}_{a},
$$

and

$$
\Phi_{1}=0 \quad, \quad \Phi_{2}=\frac{v}{\sqrt{2}}\left[H(r)+i K(r)\left(\hat{x}^{a} \sigma_{a}\right)\right] .
$$

It is well known that this ansatz for the matter fields is plagued with a residual gauge symmetry. Along with [11], we fix this freedom by imposing the axial gauge

$$
x^{i} A_{i}=0 \quad \Rightarrow \quad f_{C}(r)=0 .
$$

It is also convenient to introduce the dimensionless coordinate $x$ and mass function $\mu$ defined by

$$
x=g \frac{v}{\sqrt{2}} r, \quad \mu=g \frac{v}{\sqrt{2}} m,
$$

as well as the dimensionless coupling constants $\alpha, \epsilon$

$$
\alpha^{2}=4 \pi G \frac{v^{2}}{2} \quad, \quad \epsilon=\frac{\lambda}{g^{2}}=\frac{1}{2}\left(\frac{M_{H}}{M_{W}}\right)^{2},
$$

where $G$ is Newton's constant, $v$ is the Higgs field expectation value, $M_{H}$ is the Higgs boson mass and $M_{W}$ is the gauge boson mass. If finite, the quantity $\mu(\infty)$ defines the mass of the solution.

With these ansatz and definitions, it can be checked after an algebra that the classical equations of the Lagrangian (1) are equivalent to the equations derived from the following two-dimensional action

$$
\begin{gathered}
S=\int d t d x(-\mathcal{L}) \\
\mathcal{L}=A\left[\frac{1}{2}\left(N+x N^{\prime}-1\right)+\alpha^{2} M\right]
\end{gathered}
$$

where the prime means the derivative with respect to $x$ and the quantity $M$ is defined by 


$$
\begin{aligned}
M & =N V_{1}+V_{2} \\
V_{1} & =\left(f_{A}^{\prime}\right)^{2}+\left(f_{B}^{\prime}\right)^{2}+2 x^{2}\left(\left(H^{\prime}\right)^{2}+\left(K^{\prime}\right)^{2}\right) \\
V_{2} & =\frac{1}{2 x^{2}}\left(f_{A}^{2}+f_{B}^{2}-1\right)^{2}+\epsilon x^{2}\left(H^{2}+K^{2}-1\right)^{2} \\
& +\left(H\left(f_{A}-1\right)+K f_{B}\right)^{2}+\left(K\left(f_{A}+1\right)-H f_{B}\right)^{2} .
\end{aligned}
$$

The classical equations then reduce to the following system of six non-linear differential equations :

$$
\begin{aligned}
\mu^{\prime} & =\alpha^{2} M \\
A^{\prime} & =2 A \alpha^{2} \frac{1}{x} V_{1} \\
\left(A N f_{A}^{\prime}\right)^{\prime} & =\frac{1}{2} A\left(\frac{\partial V_{2}}{\partial f_{A}}\right) \\
\left(A N f_{B}^{\prime}\right)^{\prime} & =\frac{1}{2} A\left(\frac{\partial V_{2}}{\partial f_{B}}\right) \\
\left(x^{2} A N H^{\prime}\right)^{\prime} & =\frac{1}{4} A\left(\frac{\partial V_{2}}{\partial H}\right) \\
\left(x^{2} A N K^{\prime}\right)^{\prime} & =\frac{1}{4} A\left(\frac{\partial V_{2}}{\partial K}\right)
\end{aligned}
$$

It is important to remark that the equations are still invariant under the continuous global transformation

$$
\begin{aligned}
f_{A}+i f_{B} \longrightarrow(\exp (2 i \Omega))\left(f_{A}+i f_{B}\right) \\
H+i K \longrightarrow(\exp (i \Omega))(H+i K)
\end{aligned}
$$

where $\Omega$ is a real constant. Since the regularity of the functional $V_{2}$ at $x=0$ clearly requires $f_{A}^{2}(0)+f_{B}^{2}(0)=1$, we can fix the above symmetry by chosing $f_{A}(0)=1, f_{B}(0)=0$. This partly fix the boundary conditions which will be discussed more completely in the next section. Then, only a change of sign of the Higgs field can still be chosen arbitrarily.

Let us close this section by discussing the limit $\alpha \rightarrow 0$. From the definition (12) it is clear that the limit of vanishing $\alpha$ can be considered in different ways. If we keep $v$ fixed and let $G \rightarrow 0$ we obtain the flat limit (gravity decouples). The appropriate parameter which defines the classical energy of the solution is

$$
E_{c}=\lim _{x \rightarrow \infty} \frac{1}{\alpha^{2}} \mu(x) .
$$

It restitutes the physically-meaningful classical energy of the flat (bi-)sphaleron 2, 4, 50. In order to study the equations in the limit $v \rightarrow 0$ and $G$ fixed, then it is necessary to rescale the radial variable $x$ and mass $\mu$ according to

$$
y \equiv \frac{x}{\alpha} \quad, \quad \rho \equiv \frac{\mu}{\alpha}
$$

and to set $\alpha=0$ afterwards in the equations. We then obtain

$$
\begin{aligned}
\frac{d \rho}{d y} & =N\left(\left(\frac{d f_{A}}{d y}\right)^{2}+\left(\frac{d f_{B}}{d y}\right)^{2}\right)+\frac{1}{2 y^{2}}\left(f_{A}^{2}+f_{B}^{2}-1\right)^{2} \\
\frac{d A}{d y} & =2 A \frac{1}{y}\left(\left(\frac{d f_{A}}{d y}\right)^{2}+\left(\frac{d f_{B}}{d y}\right)^{2}\right) \\
\frac{d}{d y}\left(A N \frac{d f_{J}}{d y}\right) & =A \frac{1}{y^{2}} f_{J}\left(f_{A}^{2}+f_{B}^{2}-1\right) \quad, \quad J=A, B .
\end{aligned}
$$

In particular, the degrees of freedom $H, K$ related to the gauge field decouples and, setting $f_{B}=0$ in the equations above, the Bartnik-McKinnon (BM) equations [1] are recovered. In passing note that we have not succeeded in constructing solutions of (30) which are not related to the BM solution by mean of (25).

The corresponding energy is given by

$$
E_{B M}=\lim _{y \rightarrow \infty} \rho(y)=\lim _{x \rightarrow \infty} \frac{\mu(x)}{\alpha}
$$




\section{BOUNDARY CONDITIONS}

The regularity of the solution at the origin, the finiteness of the mass $\mu(\infty)$ and the requirement that the metric (6) approaches the Minkowski metric for $x \rightarrow \infty$ lead to definite boundary conditions (BC) for the six radial functions $\mu, A, f_{A}, f_{B}, H, K$. As far as the metric functions are concerned we have to impose

$$
\mu(0)=0, A(\infty)=1
$$

For the matter field equations two sets of BC lead to regular and finite energy solutions. [4]

\section{The sphaleron $B C$}

The flat sphaleron (and also the gravitational one) has $f_{B}(x)=H(x)=0$. The remaining functions have to obey

$$
\begin{aligned}
& f_{A}(0)=1 \quad, \quad K(0)=0 \\
& f_{A}(\infty)=-1 \quad, \quad K(\infty)=1
\end{aligned}
$$

\section{The bisphaleron $B C$}

The flat bisphalerons are characterized by the four non-trivial radial functions and we will see in the next section that they are continuously deformed by gravity. Taking into account the fixing of the global symmetry (25), at the origin the functions have to obey

$$
\begin{array}{lll}
f_{A}(0)=1 & , & f_{B}(0)=0 \\
H^{\prime}(0)=0 & , & K(0)=0
\end{array}
$$

In the limit $x \rightarrow \infty$, they have to approach constants in the following way

$$
\begin{aligned}
\lim _{x \rightarrow \infty}\left(f_{A}(x)+i f_{B}(x)\right) & =\exp (2 i \pi q) \\
\lim _{x \rightarrow \infty}(H(x)+i K(x)) & =\exp (i \pi q)
\end{aligned}
$$

So, the solutions of this type are characterized by a real constant $q \in[0,1[$; this parameter has to be determined numerically and depends on $\epsilon$ and $\alpha$.

\section{THE SOLUTIONS}

We first describe the solutions for $\epsilon=0.5$, a generic value of $\epsilon$ for which the sphaleron is the unique solution of the flat equations. The flat sphaleron is there for $\alpha=0$, with an energy $E_{c} \approx 3.64$, and gets continuously deformed for $\alpha>0$. In particular the function $N(x)$ develops a minimum which becomes deeper while $\alpha$ increases. This branch of gravitational sphalerons, let us call it $S_{l}(\alpha)$, exists up to a critical value $\alpha=\alpha_{s} \approx 0.3095$ and no solution of this type exists for $\alpha>\alpha_{s}$. However there exists a second branch of solutions that we call $S_{u}(\alpha)$ for $\alpha \in\left[0, \alpha_{s}\right]$. For fixed $\alpha$ the solution of the branch $S_{u}$ has a higher mass $\mu_{s} \equiv \mu(\infty)$ and a deeper minimum of $N(x)$ than the corresponding one on the branch $S_{l}$. This is illustrated on Fig.1; the indexes $l, u$ in $S_{l, u}$ refer to the lower, upper branch when comparing the mass.

In the limit $\alpha \rightarrow \alpha_{s}$, the solutions $S_{u}$ and $S_{l}$ converge to a common limit. It has

$$
\mu\left(\alpha_{s}\right) \approx 0.290, \quad N_{\min }\left(\alpha_{s}\right) \approx 0.513
$$


The transition from the branch $S_{l}$ to the branch $S_{u}$ is completely smooth.

In the limit $\alpha \rightarrow 0$, the solution on $S_{u}$ tends to the first solution of the BM series. This is recovered by rescaling the radial variable $x$ according to $y=x / \alpha$ and taking the limit $\alpha=0$, as explained in the previous section. The BM solution is well known but, for completeness, we present its profile on Fig. 2. It has

$$
\lim _{\alpha \rightarrow \infty} \frac{\mu(\alpha)}{\alpha} \approx 0.83 \quad, \quad N_{\text {min }} \approx 0.242
$$

We have studied the gravitating sphaleron for a few values of $\epsilon$ and determined the corresponding critical value $\alpha_{s}$. These are presented in Fig. 3. The special value $\alpha_{s}(0) \approx 0.376$ agrees with the result of [10]. We notice the rapid decrease of $\alpha_{s}$ for the low values of $\epsilon$.

We next discuss the solutions for a value of $\epsilon$ which allows both sphaleron and bisphaleron solutions to exist.

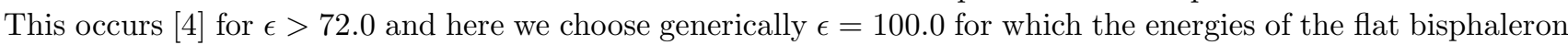
and sphaleron are given by

$$
E_{b} \approx 4.88 \quad, \quad E_{s} \approx 4.93
$$

These two solutions get deformed by gravity when $\alpha>0$ and develop two branches of solutions which we will denote $B_{l}(\alpha)$ (with mass $\mu_{b}$ ) and $S_{l}(\alpha)$ (with mass $\mu_{s}$ ). They exist respectively up to $\alpha=\alpha_{b} \approx 0.2247$ and $\alpha=\alpha_{s} \approx 0.2218$. At these critical values, we have respectively

$$
\begin{array}{ll}
\mu_{b}\left(\alpha_{b}\right) \approx 0.2080 & , \quad N_{\min } \approx 0.464 \\
\mu_{s}\left(\alpha_{s}\right) \approx 0.2047, & N_{\min } \approx 0.463
\end{array}
$$

Again, these solutions are continued by upper branches which we denote respectively by $B_{u}(\alpha)$ and $S_{u}(\alpha)$ and which coincide with $B_{l}(\alpha)$ and with $S_{l}(\alpha)$ respectively at $\alpha=\alpha_{b}$ and $\alpha=\alpha_{s}$. The two bisphaleron-solutions corresponding to $B_{l}$ and $B_{u}$ for $\alpha=0.2$ are presented on Fig. 4.

All along the two branches the energy of the gravitating bisphaleron stays slightly lower than the one of the sphaleron. Of course both quantities become equal when $\alpha=\alpha_{c}$, this is illustrated by Fig. 5. This figure also clearly indicates that, at the critical value $\alpha_{s}$ (resp. $\alpha_{b}$ ), the energies of the two gravitating sphaleron $S_{l}, S_{u}$ (resp. bisphaleron $B_{l}, B_{u}$ ) solutions form a cusp. When bisphalerons are present, four solutions are available on some interval of $\alpha$ and there are two cusps.

Completely similarly to the case $\epsilon=0.5$, the branch $S_{u}$ converges to the Bartnik-Mckinnon solution in the limit $\alpha=0$. The scenario with the branch $B_{u}(\alpha)$ is different: decreasing $\alpha$ from $\alpha_{b}$ we observe that the different radial functions composing this solution uniformly approach their counterparts of sphaleron solution $S_{u}$. This occurs for $\alpha \approx \alpha_{c} \approx 0.185$ as illustrated on Fig. 6 . On this figure the quantities $f_{B}(\infty)$ and $H(0)$ which characterize the bisphaleron are plotted for the different branches. The minimal value $N_{\min }$ of the function $N(x)$ is superposed on the figure.

In view of these results we can say that the branch $B_{u}$ of solutions bifurcates from the branch $S_{u}$ at $\alpha \approx 0.185$.

In order to have a qualitative idea of how the gravitating (bi)-sphaleron solutions behave for higher values of $\epsilon$, we solved the equations for $\epsilon=800.0$. There the gravitating sphaleron and bisphaleron exist respectively up to $\alpha_{s} \approx 0.209$ and $\alpha_{b} \approx 0.221$. The branch $B_{u}$ bifurcates from $S_{u}$ at $\alpha \approx 0.07$

This suggests that, when $\epsilon$ increases, the branch $B_{u}$ bifurcates from $S_{u}$ for lower values of $\alpha$ and that the gravitating bisphaleron exists on an interval which becomes relatively larger than the interval of existence of the sphaleron.

In the limit of the non-linear sigma model $\epsilon=\infty$, it is known [2,4 5 ] that the sphaleron is discontinuous at the origin unlike the bisphaleron which continues to exist as a regular solution. We expect that in this case the bisphaleron branch $B_{u}$ will exist up to $\alpha=0$ where it will approach the Bartnik-McKinnon solution.

Let us finally say some words about the stability of the various solutions. For $\epsilon<72.0$ the flat sphaleron possesses a single direction of instability (a negative mode) and for $\epsilon>72.0$ the sphaleron has two directions of instability while the bisphaleron has one 15.13 . In this respect the sphaleron (resp. bisphaleron) is interpreted as the minimal energy barrier [2] bewteen topologically different vacua of the Weinberg-Salam model for $\epsilon<72.0$ (resp. $\epsilon>72.0$ ).

The shape of the plot of the energy, with the two branches terminating into a cusp is typical for catastrophe theory (see e.g. [12]) and the use of arguments based on Morse theory suggests many useful information about 
the stability of the different solutions. For instance the number of negative modes for the solutions on the upper branch exceed by one unit the number of negative modes for the solutions on the lower branch. Such a reasonning was demonstrated to be correct e.g. in [14].

Using the same arguments in the present context indicates that the solutions on the branch $S_{l}$ of Fig. 1 have one direction of instability while the solutions of the branch $S_{u}$ (and therefore also the BM solution) have two.

For the solutions of Fig. 5 we have one (resp. two) directions of instability for the solutions on $B_{l}$ (resp. $B_{u}$ ) and two directions of instability for the solutions on $S_{l}$. On the branch $S_{u}$ the number of negative modes is equal to two on the interval $\alpha \in\left[0, \alpha_{c}\right]$ (i.e. before the branch $B_{u}$ has bifurcated) and equal to three for $\alpha \in\left[\alpha_{c}, \alpha_{s}\right]$ (i.e. after the bifurcation). The number of instable modes at the approach of the BM solution is then equal to two, irrespectively of the parameter $\epsilon$.

Obviously these deductions would need to be confirmed by more elaborated calculations.

\section{CONCLUSION}

The coupling of the Yang-Mills-Higgs equations to gravitation often leads to interesting new properties of the available gravitating classical solutions [15, 16].

The classical equations of the Weinberg-Salam model possess a rich pattern of bifurcations of bispahleron solutions [4,5] from the sphaleron solution [2]. It is therefore natural to attempt to study the critical phenomenon which occur in the Einstein-Weinberg-Salam equations. In response to gravity, parametrized by the quantity $\alpha$ defined in (12), the equations exhibit another type of critical phenomenon : the occurence of two branches of solutions which terminate at a critical value of $\alpha$; this was noted in 10 for $M_{H}=0$ but the phenomenon occurs for generic values of $M_{H}$.

Here we were interested only in global solutions (i.e. the metric function $N$ has no zero on $[0, \infty]$ ), but another interesting feature of gravitationally deformed classical solutions (soliton or sphaleron) is the existence of black hole solutions [15] where the function $N(r)$ develops an horizon at some finite value $r=r_{h}$, i.e. $N\left(r_{h}\right)=0$. We guess that flat (bi-)sphalerons could also be deformed in this way and produce sphaleron black holes.

[1] R. Bartnik and J. McKinnon, Phys. Rev. Lett. 61 (1988) 141.

[2] F.R. Klinkhamer and N.S. Manton, Phys. Rev. D 30 (1984) 2212.

[3] G. t'Hooft, Phys. Rev. Lett. 37 (1976) 8.

[4] J. Kunz and Y. Brihaye, Phys. Lett. B 216 (1989) 343.

[5] L.G. Yaffe, Phys. Rev. D 40 (1990) 3463.

[6] N.S. Manton, and T.M. Samols, Phys. Lett. B207 (1988) 179.

[7] Y. Brihaye, S. Giller, P. Kosinski and J. Kunz, Phys. Lett. B293 (1992) 383.

[8] Y. Brihaye and T. Tomaras, Nonlinearity 12 (1999) 867.

[9] B.R. Greene, S.D. Mathur and C.M. O’Neill Phys. Rev. D47 (1993) 2242.

[10] M. S. Volkov and D. V. Gal'stov, hep-th/9810070.

[11] T. Akiba, H. Kikuchi and T. Yanagida, Phys. Rev. D38 (1988) 1937.

[12] F. V. Kusmartsev, Phys. Rep. C183 (1989) 1.

[13] Y. Brihaye and J. Kunz, Phys. B249 (1990) 90.

[14] Y. Brihaye, J. Kunz and C. Semay, Phys. Rev. D42 (1990) 2846.

[15] P. Breitenlohner, P. Forgacs and D. Maison, Nucl. Phys. B343 (1992) 357, ibid, B442 (195) 126.

[16] A. Lue and E.J. Weinberg, Phys. Rev. D60 (1999) 084025. 


\section{Figure captions}

Fig. 1.

The mass of the gravitating sphaleron solutions (represented by $\mu_{s}$ and by $\mu_{s} / \alpha$ ) and the minimal value $N_{m}$ of $N$ are reported in function of $\alpha$ for $\epsilon=0.5$.

Fig. 2.

The profiles of the functions $N, A, f_{A}$ of the solution $S_{u}$ as functions of $y=x / \alpha$ for $\alpha=0.01$ and $\epsilon=0.5$.

Fig. 3.

The critical value $\alpha_{s}$ as a function of $\epsilon$ for the low values of $\epsilon$.

Fig. 4.

The profiles of the functions $N, A, f_{A}, f_{B}, H, K$ of the bisphalerons $B_{l}$ (in dotted) and $B_{u}$ (in solid) for $\alpha=0.2$ and $\epsilon=100.0$.

Fig. 5.

The masses $\mu_{s}, \mu_{b}$ of the gravitating sphaleron (solid) and bisphaleron (dotted) as functions of $\alpha$ in the region of the critical value. The branch $B_{u}$ stops at $\alpha \approx 0.185$, as indicated by the star.

Fig. 6.

The values of $H(0), f_{B}(\infty), N_{m}$ for the gravitating bisphaleron in function of $\alpha$. The corresponding value of $N_{m}$ for the sphaleron is represented by the dotted line. 
Figure 1

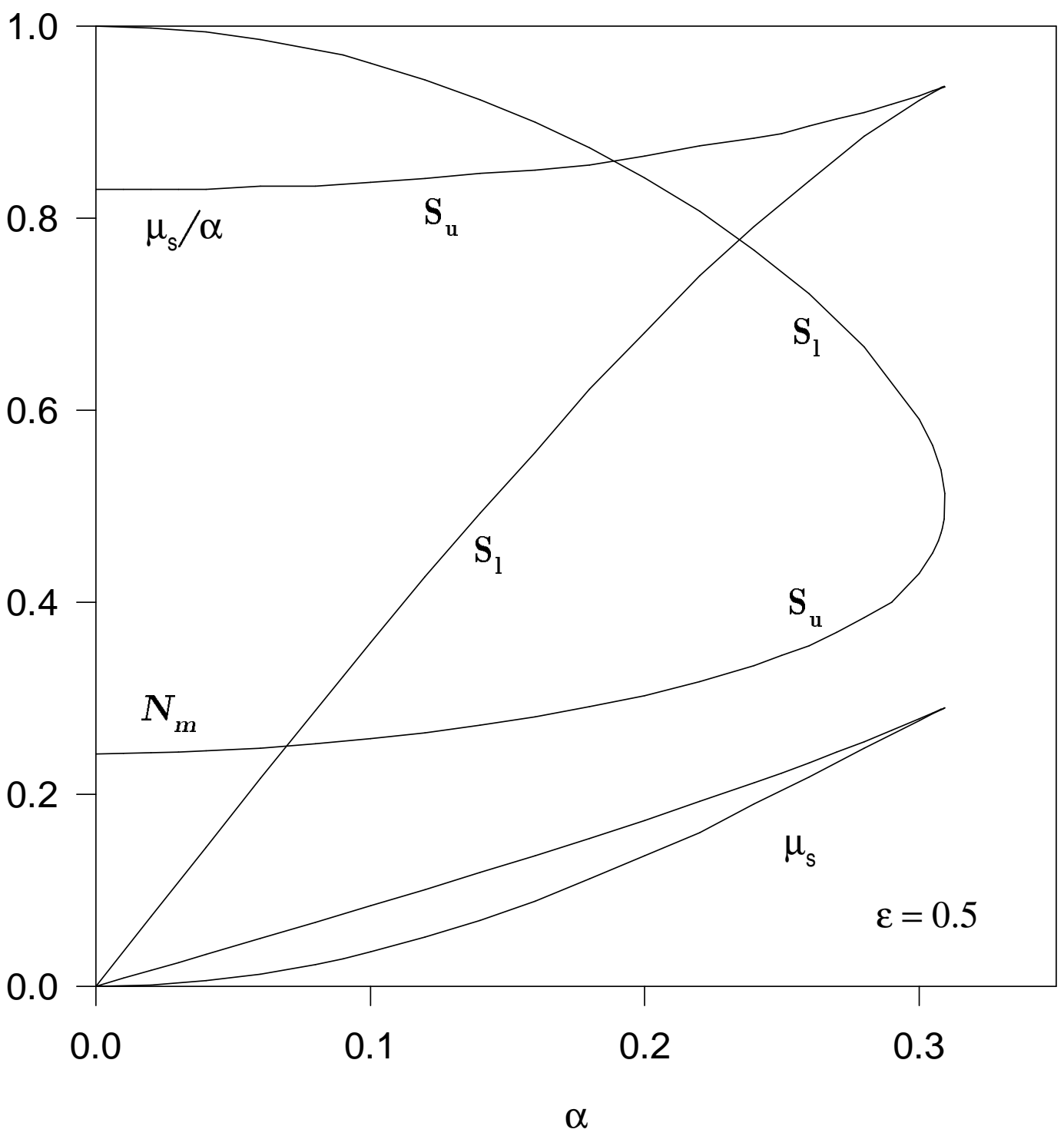


Figure 2

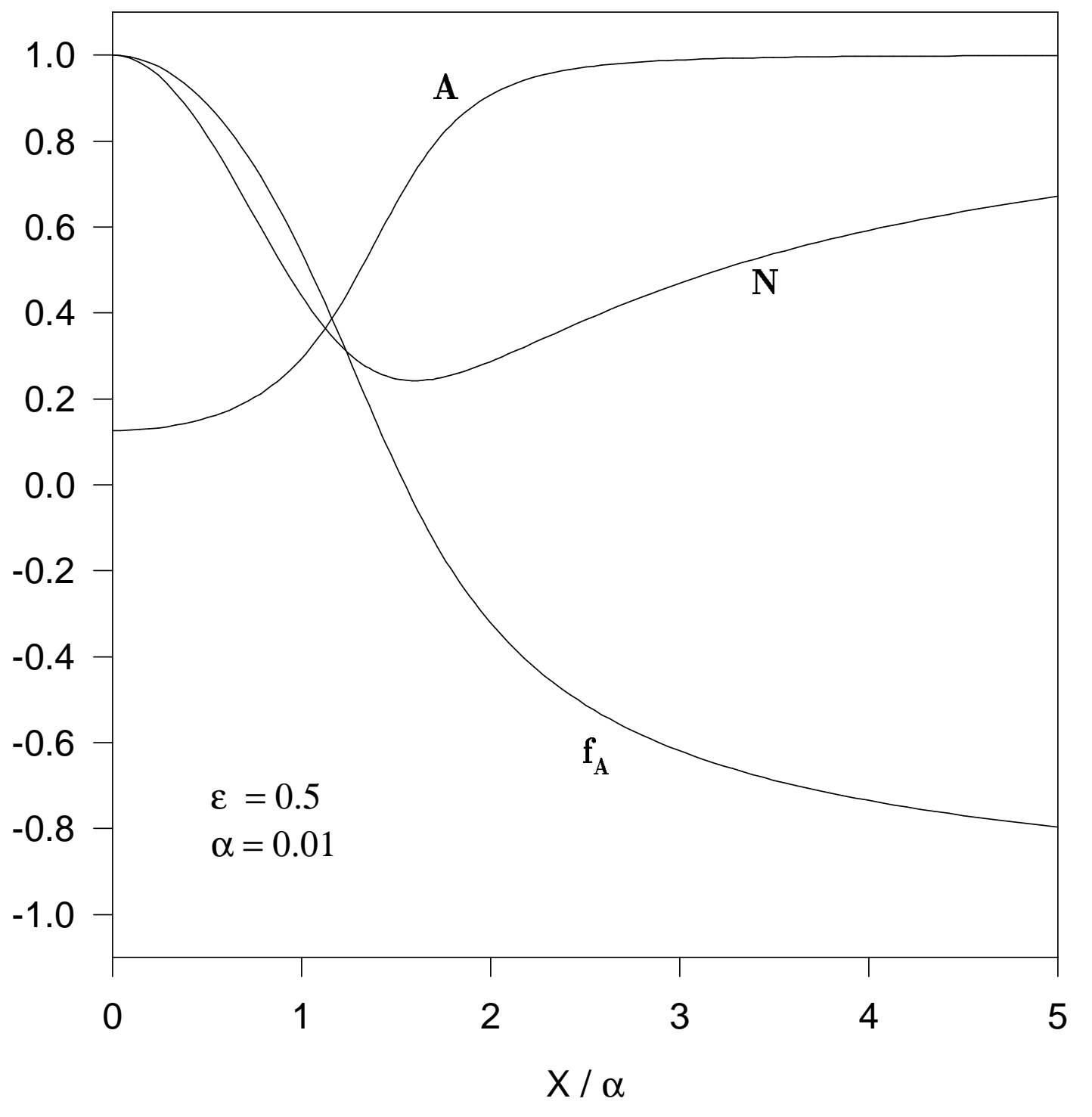


Figure 3

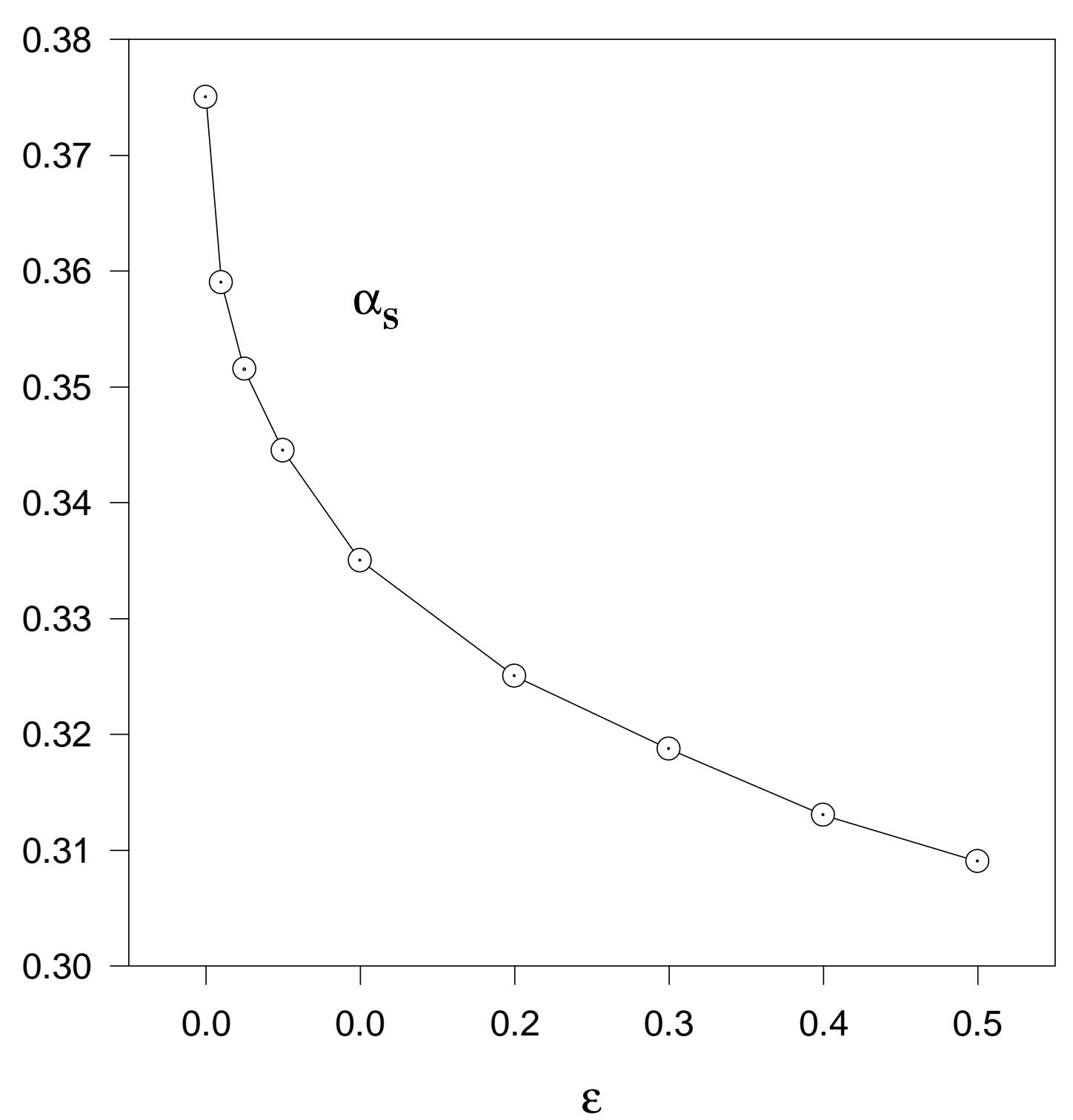


Figure 4

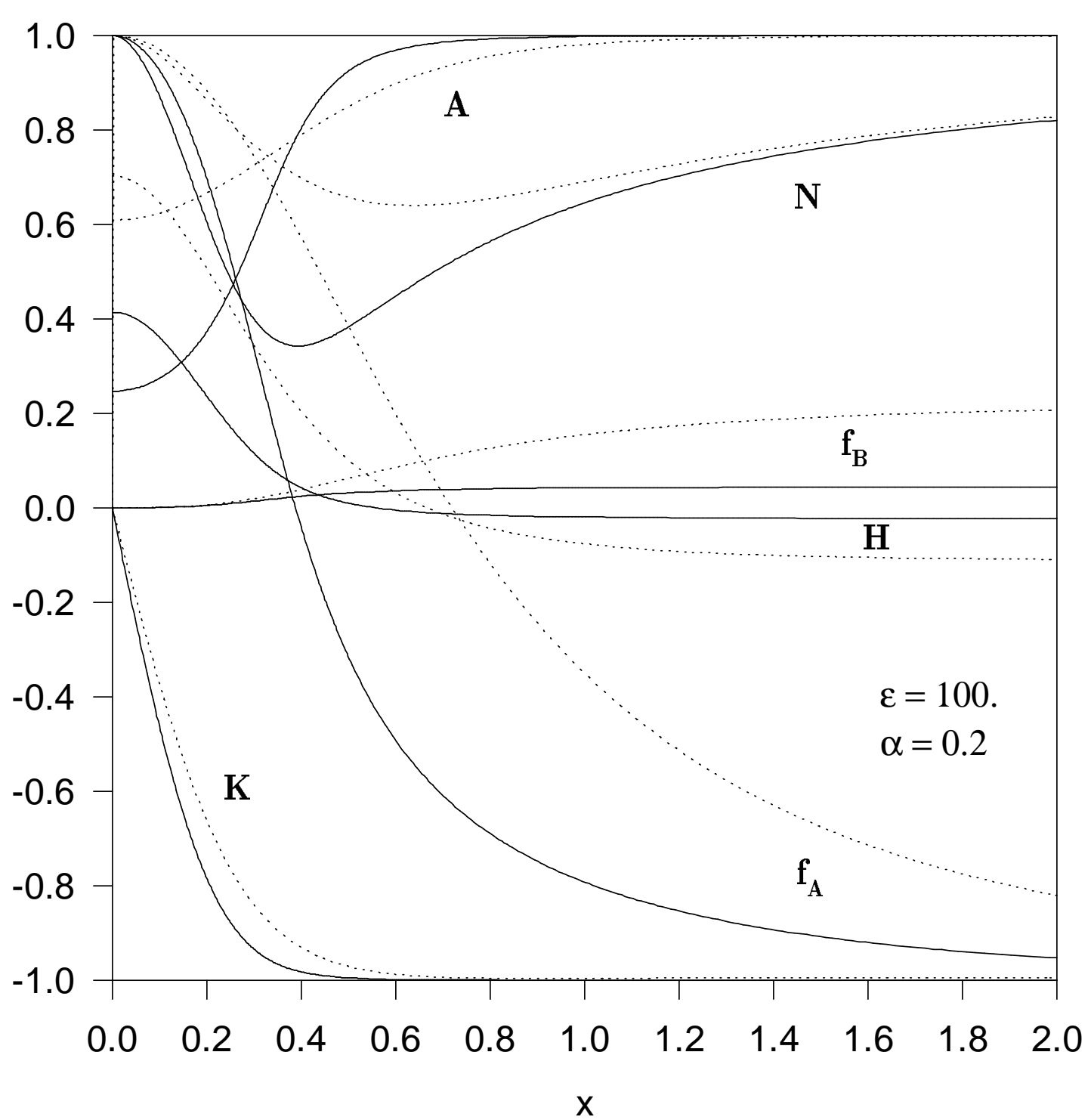


Figure 5

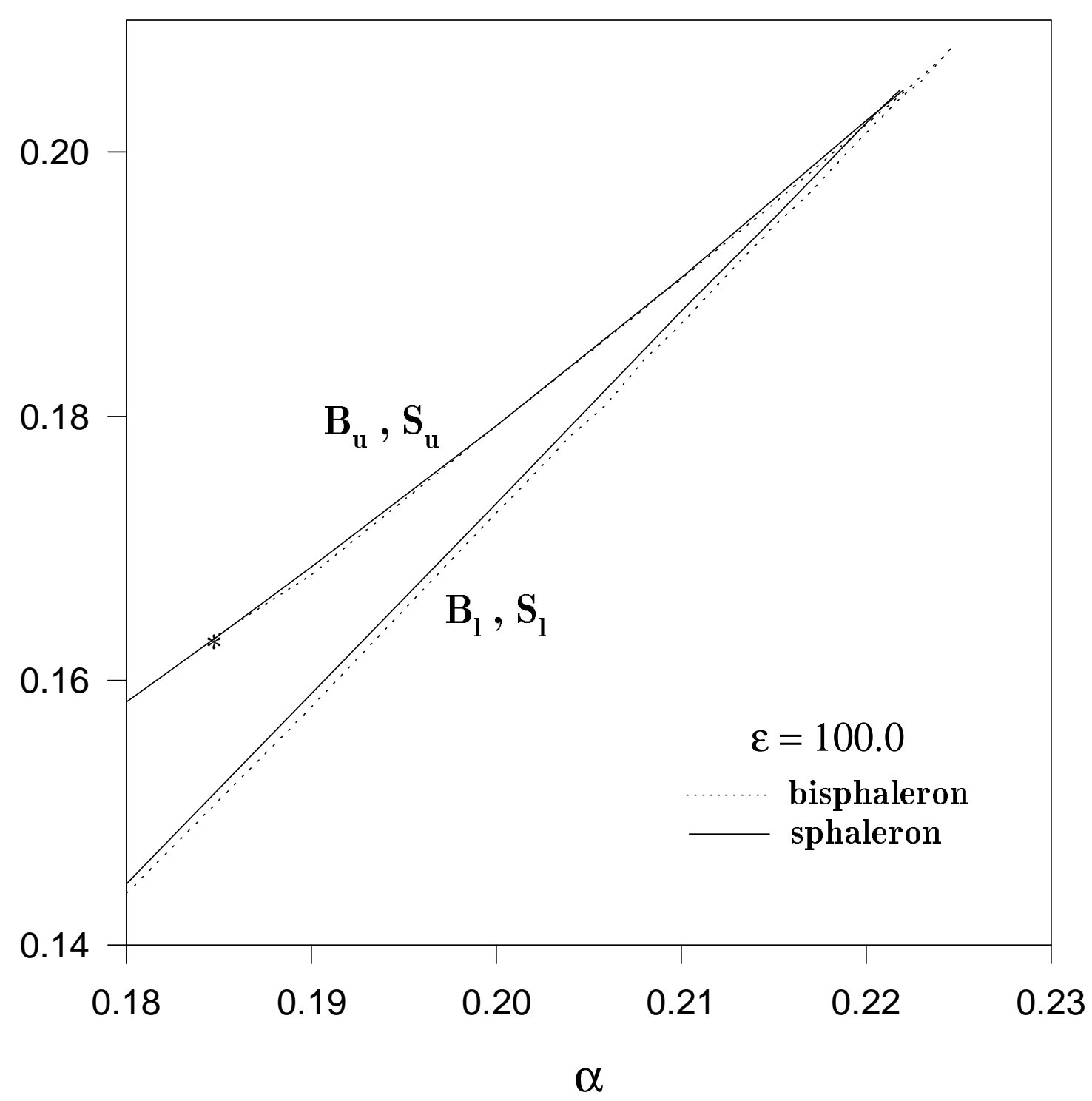

